\title{
$\mathfrak{I} \mathfrak{n} \mathfrak{h} \mathfrak{a} \mathfrak{l} \mathbf{t}$
}

\section{gifloriote fieter.}

1. Theatrum mundi. T. i. comỏbietoeife bar= geftellte Begebenbeiten, fo in Den Jabren Ghrifti unfers ફ̧erm 1653-1657 in Eumm ibren Bor=

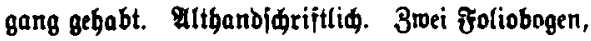
5ofprediger Jacobi in Sloburg gehörig. 1831.

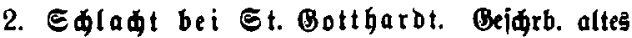

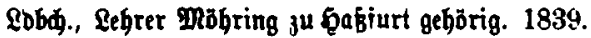
Die Ueberifrift hieß bort: Bcidlagener ₹ürth .

3. ङ中la bt bei $€ t$. Bottharbt. Fin gang newes Lieb ibber bas Frmiftitium io Der Brobmedtigfte Ravier Reopolbus I. mit bem türtijien Erbjeinbt

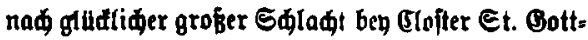

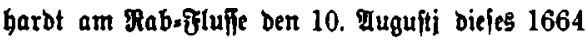
Jabre auff 20 Jahr abgeldlofien. 4. 2 gal. o. 3 . In ber Erbidajtsmā̄e ber Żamilic v. Rretiळmann zu Cbertheres. 1830 . . . . . . . . . 14

4. Beiprád jwifden England und Rubter. altes శiolioblatt, Brofeffor Tentinger in $\mathfrak{R}_{\text {ür }}=$ burg gebörig. 1832. Wabrideinlid fehlt nod etroas, Denn bie andere Gălfte bes Bogens ră abgerifien. leber/drift nimt borhanden 


\section{VIII}

$\therefore$ Mgeinfelden. 3weg lä̆ne Rewe dieber. Tas Erfte: Bon Golmar. Colmar, wo willt bu jegt bin 2c. Das Andere: Bon Mgeinjelden. Der Mhein=Brafi und ber Edybede as: Im Эahr Chrifti, 1675. 8. 4 BL. D. O. Berlin $\mathfrak{D}_{e} 7686$ unb De 7771 . . . . . . . . . . . . .

6. Belägerung Mbeinfelos. Mbeinjelder He=

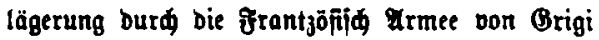

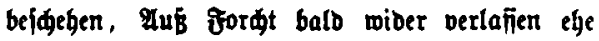
ifn $39 x$ Turdl. Don Lotgringen redt tonnen ars fafien. 8. \& gal. o. Э. u. L. mit Kitelbolz= ifnitt . . . . . . . . . . . . . 24

7. Straß̧итg. Staatzbibl. зи 9Ründen. Cod. germ. p. 136-137 . . . . . . . . . . 29

8. Sकla巾t bei patras. Feltere bano= i出rift . . . . . . . . . . . . . . 36

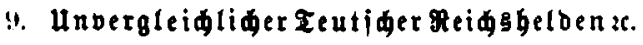
qute פanbidrift . . . . . . . . . . . 39

10. Confessio Gall ica na. Staaţbibl. zu Mtinden. Corl. gern. 4055. p. 165-173 . . . . . 47

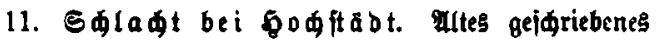
Sieberbu山 . . . . . . . . . . . 56

12. Im Quartiere. Dessgleiden . . . . . . 58

13. Belagerung von Toulon. Şandidriftlid aus jenter 3eit . . . . . . . . . . . 60

14. Sめladt bei Dlalplaquet. Des̆gleiden . . 61

15. Sanz neues welttbeater. 8. 4 Bal. D. D. 63

16. Beter des BroBen Tod. Ganbidriftlid aus iener 3eit . . . . . . . . . . . . . . . . . . 69

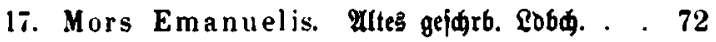




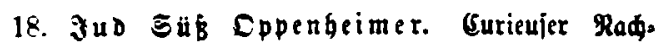
riфten. Hus Dem Meid Der Bejdnittenen. Erfte Unterredung, Jwij内en Sabatbai Sebi, Einem in

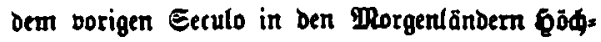
berildtigt getwijenen Эiubija: Grbetrüger, Und Dem fameujen $\$$ Jojepg Sü Cppenteimer z. Erftes Etüd. Be. brưt zu Cana in Balilāa. 1737. 1 . . . 74

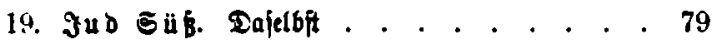

20. Ter wagrbajte 3eitungsiinger. Gandidrijt jener 3eit . . . . . . . . . . . . . 84

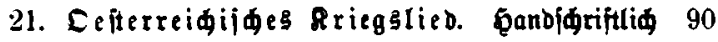

22. $\Re$ ädzug berer franzolen bon Prag. Gand. idrift jener 3eit . . . . . . . . . . . 92

23. Belagerung ber Beftung Rottenberg. Danbjurift jener 3eit . . . . . . . . . 94

24. Sarl Mlbert's Tob. Bejurb. Bobd. jener Beit 99

\section{Balladen.}

25. F a uft. Mtüblid in Unterfranfen. 1832.105

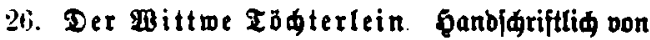

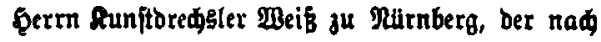
Dem Boltsmunde bas Sied aufjeifnete. . . . 110

27. Ein Traum. Befdrb. Sbbb. aus Raftell in Unterfranten. 1832 . . . . . . . . . 112

2x. Saniel unb Bretel. Bejdrb. Sobb. bon 1691 aus Ritïingen. . . . . . . . . . . . 115

29. Ditller und Bauer. Relteres gejorb. Bobd. aus Edweinfurt . . . . . . . . . . . 117

30. Das fette S中weinlein. Befdrb. Sbbd. aus kömershofen . 
31. જrommet \$ุund. Qejorb. Lobd. aus greft: bein 1833 . . . . . . . . . . . . 125

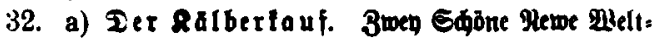

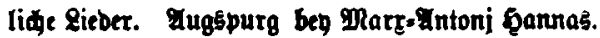

8. 4 Ba. o. 3. . . . . . . . . . . . 1.88

32. b) $I a g$ Regger $=\&$ ieb. Reue fuftige und fiderj: baite sieber. 8. 2 9ll. o. 9. u. . . . . . 131

33. Cupido uno ber Clausner. Flte פandidrift 134

34. Japonia septentrionalis. altes geidrb. Sobd. . . . . . . . . . . . . 137

35. Die Edneider und bie Beis. Beidrb. alteres gbbd. . . . . . . . . . . . . 141

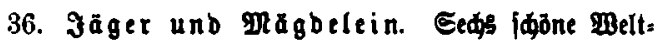
lide Rieber. 8. 4 Bal. o. 3. u, C. . . . . 144

37. Der Bettler. Mlte நanbidrift . . . . . 147

38. Iraum. Seds neue Meltlide Rieber. 8. 4 Bll.

D. 9. u. D. . . . . . . . . . . . . 149

39. Bemedt. Mnunblid, in Unterfranten. . . . 151

40. Der Erganift. Ganbidriftid, aus bem Enbe Deg sorigen Jabrh. . . . . . . . . . . 153

41. Bebentlid. פeunblid, in Nämberg . . 157

42. Das Oretle von Strimpilebad. Müblid, aus ber Begend bon Meutfingen . . . . . . 15x

43. In gefu h $\mathrm{rt}$. Detublid, aus b. Begend b. Dillingen 161

44. Siebesgefprăd cines Eranzolen. Eolbaten= unb andere jđöne Rieber. 8. 2 \&u. o. 3. u. . . 163

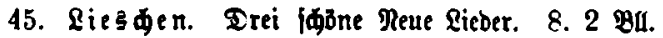
о. 3. u. C. . . . . . . . 160;

Sicbeslieder.

46. Rebrt um! RItes geidrh. Robd. nue bolgeim. 1834 . 
47. Irauer. Der ander Theyl, Sqöner turbmeiliger

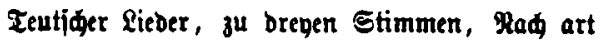
Der Reapolitaner sc. netwlid Durd stöm. Rec. May. Ptuficum, Jacobum Regnart, componirt zc. Ruirnberg, 1578. \$r. 31 . . . . . . . . 173

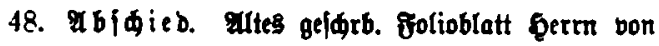
Gomthal in Bamberg gehorig. 1837 . . . . 175 49. Die Graujame. Ganb/driftlid. . . . . 17i 50. Ibldiebsidreiben an cinen Beliebten. 8. 2 pu v. 9. u. . . . . . . . . . . 178

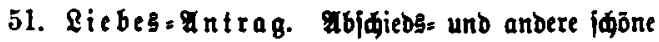
Lieber. 8. 2 gll. o. 3. u. C. . . . . . . 181

52. Unbefieglid. Bejurb. Sobd. . . . . 183 53. $\mathscr{A}_{\mathfrak{n}} \Theta_{\mathfrak{q} l \text { bia. }}$ Dajelbft . . . . . . . 185

54. Dod treu. Tajelbft. . . . . . . . . 187

55. Gieg ber Beftändigteit. Tajelbit . . . 190

56. Die Diebin. Ganbjuriftlid . . . . . . 193

57. Siebegphantajien. Bejdrb. SDbd. . . 194

58. Weidit, quälende Bebanten. Melteres geidrtb. Sobd.

59. A b出 i e ๖. J. u. . . . . . . . . . . . . . . 194

60. Treue \&iebe. Altcs geldrb. \&obd. . . . . 202

61. Siebesfdmerzen. Befdrb. Mufitheft . . . 204

62. Mbidied bon ber Beliebten. Mundlid, in mürburg . . . . . . . . . . . . . 206

63. Eo jind die Mäbden. 8. 2 \&u. o. 3. u. C. 208 64. Lotthen. 8. 2 Ba. o. Э. u. C. . . . . . 209 65. ₹ruke nur! Mundi俩, in unterfranten . . 211 66. Ein Rind bon zranzig gagren. Pundid, in ber Begend von mutzburg . . . . . 21 


\section{XII}

Eette

67. Treue \&iebe. Münblid, in ber Gregent von Diflingen . . . . . . . . . . . . 216

68. 5ุang $\mathfrak{Q} \mathfrak{u} \mathfrak{f t}$. . . . . . . . . . 218

\section{veruildte eicder.}

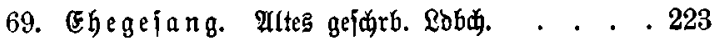

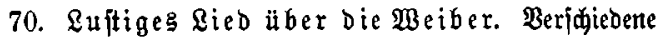
¡đöne neue Lieber. 8. 2 BH. о. Э. น. D. . . 225

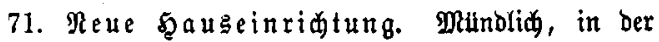
Begend von Regenģurg . . . . . . . . 227

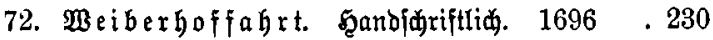

73. Der Bauer als Refrut. Ultes gefirb. Robd. 234

74. Bauernfelozug. 2lites gejđrb. Babd. . . . 237

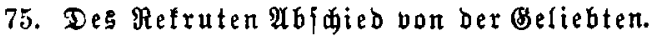
Mindilid, in Unterfranfen. . . . . . . . 241

76. Der Dejerteur. Mündlid, in Der Begend von Eanbeghut . . . . . . . . . . . 243

77. Ein Эäger will id werben. Mütblid, in Unterfranfen . . . . . . . . . . . . 248

78. Der $\mathfrak{B}$ ildjulats. Mündlid, in ber Begent von Zrcifing . . . . . . . . . . . . . . 251

79. Der Mild fidig. Müblid, in ber Gegend von Lanbghut . . . . . . . . . . . . . 254

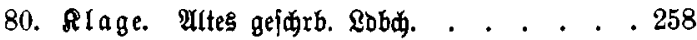

81. Beruhigung. Dajelbft. . . . . . . . 260

82. Blajie Sorgen, gute Radt! Dajelbit . 262

83. Biber ben Sdlaf. Dajelbft . . . . . 265

84. Sqofinungalog. Dajelbft . . . . . . . 268

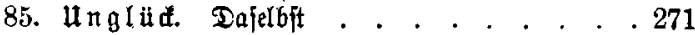

86. Beftänbigfeit. Dajelbjt . . . . . . . 274

87. Beftändigfeit. Dajelbit . . . . . . . 276 


\section{XIII}

Ecire

88. Gofinung und Ireue. Tajelbit . . . . 27!

89. Wie mir's gefältt. Sandidriftlid . . . 281

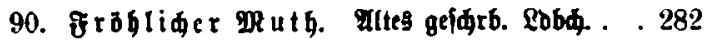

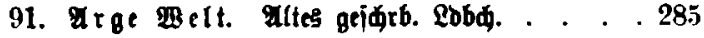

92. Sleinmutb. Dajelbft. . . . . : . . 288

93. Rein Gelb. ultes gejurb. Sbbh. ans ber

Cegent bon Tmberg . . . . . . . . . 290

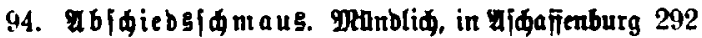

95. Der Eabrbeitsfreunb. Eltes gejurb. Sobd. 294

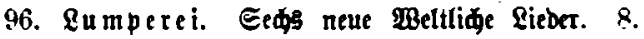
4 BII. o. Э. u. E. . . . . . . . . . . 294

97. Das Bettelleutlieb. Solbaten= unb anbere

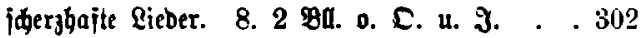

98. Ter luftige Ergelmann x. 8. 2 Ra. o. Jे.

u. E. . . . . . . . . . . . . . 305

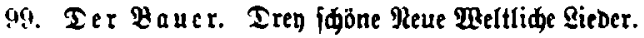

Das Crfite. Bon ben grofien ఏelo Zrieberid, wic ign bie Staroften eridofien baben zc. Das Fnbere.

I4 bin ein freber Bauerstnedt zc. Das irittc. $D$ bu Jdmarzes Meuglein ad! zc. Bebnud in Diejem Jabr. 8. 4 Bu. o. Э. u. S. . . . . 311

1no. Der Paucr. Btweb Sböne Retwe Rurbmeilige Lieber. Das Erite: Mein Batter ift tein Ebel: mann sc. Tas Anber: Gin Rrieg bat fid gefangert an 2. . $3 \mathrm{~m}$ Zhon: Wie man ben

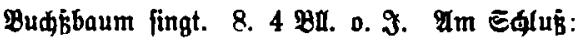
Bebruat ju Æugipurg, beb (56riftoif Sdmio . 31;

101. \$̨offärtige 3eit. Melteres geidrb. Sobd. . 318

102. Per Banerufnedt. Müblid, in ber Cegeno von Meuburg an ber Donau . . . . . . . 320

103. Eridaffung ber $\mathfrak{W}$ elt. Wltes Rieberhert aus

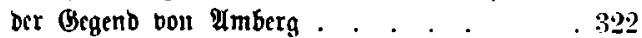




\section{XIV}

104. Petrus. Dajelbft . . . . . . . 337

105. 28cignadtslieb. altes geidrb. 8Dbd. . 340

106. Unfer liebe ơ rau 子u Bersbad. Dajelbit 343

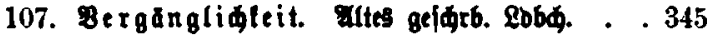

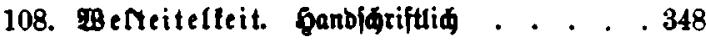

109. Der Tob. Flte Ganbidrift. . . . . . 350

110. Der treue \$ilot. Ganbldrift bom Jabre 1792352

111. Des bl. Rirdenlebrers Auguftini Bort bei bem rab C. Julii Caesaris etc. Uhi nunc est tua magnificentia Caesar? Threnodiae beatis Manibus Viris magnifici, nobilissimi Dm. Johannis Jacobj Tetzelii a Kirchsittenbach. Norimbergae 1646 . . . . 357

\section{Angang.}

112. Bilimer. Boetifde Cyren bes erbart uno Wolvornemen berrn Boligang Watius Butbrobs 2 . Purmberg 1649389

113. Ingefodtene freibeit. Wundner Staats: bibliotbet, C. germ. 4395, b. c. d. e. . . . 355

114. Berfodtene freigeit. Dafelbft . . . 365

115. Domine, quid me vis facere? Gandifriftl. 371

116. Fin andadtiges Bater:llnier. In honorem maledicti officjalis Dechelj. Ieltere ఏanb/đrift 380 